\title{
Why Using Age as a Proxy for Testosterone is a Bad Deal
}

\author{
Felix D. Schönbrodt \\ Department of Psychology \\ Ludwig-Maximilians-University Munich, Germany
}

\begin{abstract}
In their article "Deal or No Deal: Hormones and the Mergers and Acquisitions Game," Levi, Li, and Zhang (2010) claimed that they investigated the effect of testosterone on CEOs' decisions in mergers and acquisitions. However, they did not measure testosterone levels directly. Rather, they tried to use CEO age as a proxy, based on a previously documented negative correlation between age and testosterone level. In this comment, I argue that it is not reasonable to use age as a proxy for testosterone, and that Levi et al.'s study does not tell us anything about testosterone. General remarks on using proxy variables are given.
\end{abstract}

In their article "Deal or No Deal: Hormones and the Mergers and Acquisitions Game," Levi, Li, and Zhang (2010) investigated the research question of whether the hormone testosterone ( $\mathrm{T}$ ) has an impact on decisions in mergers and acquisitions (M\&As). Based on experimental results that $\mathrm{T}$ has an effect on behavior in ultimatum games (Burnham, 2007), Levi et al. hypothesized that CEOs with higher $T$ levels should show more aggressive/dominant behavior in M\&As. To investigate this hypothesis, the authors assembled a data set with $357 \mathrm{M} \&$ As and several economic variables related to them (e.g., the size of the target firm, the board size, and several other economic indicators). As they could not assess the $\mathrm{T}$ levels of the CEOs directly, they "[...] have suggested an alternative: specifically, to proxy testosterone by age" (p. 1476). Therefore, as the authors admitted themselves, their reasoning was based on a central assumption: "The validity of this approach clearly depends on the extent of the association of hormone levels with age." (p. 1476). To summarize their findings, a significant but small effect of age on M\&A decisions was found: younger CEOs made more bid withdrawals and more tender offers than their older counterparts (which has been interpreted as more dominant behavior). As the story has received wide press coverage, for example, in the Wall Street Journal, Financial Times, Time Magazine, and the Los Angeles Times, I feel the need to make some clarifications.

Empirically, Levi et al. have shown an effect of CEO age on the outcome of M\&As. From the title to the conclusion

Felix D. Schönbrodt, Department of Psychology, LudwigMaximilians-University Munich, Germany. I want to thank Birk Hagemeyer for valuable comments on a previous version of this comment. Correspondence concerning this article should be addressed to Felix Schönbrodt, Leopoldstr. 13, 80802 München, Germany. Email: felix.schoenbrodt@psy.lmu.de of their article, however, they refer to the effect of testosterone (e.g., "[...] in M\&As the testosterone of both parties could influence the course and outcome of negotiations," p. 1463; "[...] we consider whether testosterone influences the likelihood that offers made are subsequently withdrawn," p.1466; "This finding strongly supports an association between testosterone, as proxied by the bidder male CEO age, and M\&As," p. 1469).

In the following commentary, I argue that it is not appropriate to use age as a proxy for $\mathrm{T}$ level and that the conclusions of Levi et al. are taking it way too far. For the clarity of my arguments, I will focus only on the strongest reported effect. For all weaker effects, the same reasoning applies even more.

\section{The Effect of Testosterone on Dominant Behavior is Rather Low}

Is it a reasonable hypothesis to expect more dominant M\&A behavior from CEOs with higher T levels? Early investigations with animals have shown a relation between testosterone level and aggressive or dominant behavior (e.g., Wingfield, Hegner, Dufty, \& Ball, 1990). Recent review articles and meta-analyses on human testosterone, however, have shown that the effects in humans are rather small. For example, in a meta-analysis on the relation of male human aggression and $\mathrm{T}$ level ( $\mathrm{N}=9760$; Archer, GrahamKevan, \& Davies, 2005; Book, Starzyk, \& Quinsey, 2001), the weighted correlation was only.08. According to Cohen (1992), this is a trivial effect size. Other meta-analyses on the relation of $\mathrm{T}$ to dominance (weighted $r=.13$ ) and to a challenge in a sports competition (weighted $r=.18$ ) have supported this finding of a small effect (for an overview, see Archer, 2006). To summarize, if M\&As are seen as competitive situations, indeed, an effect of $\mathrm{T}$ could be expected - but the relation is probably much less pronounced than common sense might suggest. 
F. SCHÖNBRODT

\section{The Relation Between Age and Testosterone is Rather Low}

The central assumption in their article is that $\mathrm{T}$ level can be proxied by age. How strong is that relation? A metaanalysis of 23 studies reporting the correlation between age and $\mathrm{T}$ level $(\mathrm{N}=1900)$ revealed that the average correlation between these variables was -.18 (Gray, Berlin, McKinlay, \& Longcope, 1991). That means only $3 \%$ of the variation in $\mathrm{T}$ level can be explained by age. Levi et al. refer to another study that shows a remarkably stronger correlation of -.50 (Harman, Metter, Tobin, Pearson, \& Blackman, 2001). Even with this value, $75 \%$ of the variation in $\mathrm{T}$ remains unexplained by age.

All articles cited by Levi et al. report the correlation for a broad age range (e.g., 24 - 90 years, Ferrini \& BarrettConner, 1998; 23 - 91 years, Harman et al., 2001). Given that $90 \%$ of CEOs in Levi et al.'s sample were between 46 and 64 , a massive range restriction is present, which presumably lowers the correlation in that age range even more. Indeed, the scatter plots in Harman et al. (2001) strongly suggest that the correlation is mainly driven by the very young and very old participants. But regardless of whether the true correlation is closer to -.18 or to -.50 : Are these correlations high enough that age is warranted as a valid proxy for T level? As we will see in the next section, the answer is no.

\section{A "Triangulation" of an Unobserved Correlation?}

In their conclusions, the authors argue as though they had established a causal effect of T on M\&A decisions. However, they had not even established a correlation between $\mathrm{T}$ and M\&A behavior. Their approach seems to be something like a "triangulation" of an unobserved correlation by the knowledge of two other correlations. Indeed, there are dependencies and constraints in the relation of the bivariate correlations between three variables. If two of the three correlations are given ( $r_{13}$ and $\left.r_{23}\right)$, the possible range of the third correlation, $r_{12}$, is constrained by following equation (Olkin, 1981):

$r_{13} r_{23}-\sqrt{\left(1-r_{13}^{2}\right)\left(1-r_{23}^{2}\right)} \leq r_{12} \leq r_{13} r_{23}+\sqrt{\left(1-r_{13}^{2}\right)\left(1-r_{23}^{2}\right)}$

Figure 1 graphically represents the relationship of variables given by this equation. The left plot shows the upper boundary of $r_{12}$, the right plot the lower boundary of $r_{12}$. As one can see, rather high values for either $r_{13}, r_{23}$, or both, have to be present to imply a positive sign (i.e., a lower boundary $>0$ ) of $r_{12}$.

Given the observed estimates of $r_{\text {age }, T}=-.50$ and $r_{\text {rage,bid }}$ $=-.12$, the range of possible values for $r_{T, \text { bid }}$ goes from -.80 to +.92 . (The position is marked by the asteriks in Figure 1.) If the probably more realistic estimate from the metaanalysis is used ( $r_{\text {age }, T}=-.18$; Gray et al., 1991), the possible range for $r_{T, b i d}$ goes from -.95 to +1.00 . These calculations clearly show that there is no argument to expect a positive correlation between $\mathrm{T}$ and M\&A decisions. Actually, with the given data set, no conclusions about $\mathrm{T}$ can be drawn at all.

\section{The Effect of Age on M\&A Decisions: An Artefact?}

Given these analyses, it should be clear that one cannot speak of a $\mathrm{T}$ effect based on these data. What about the age effect reported in this article? The authors wrote that, "[... $\mathrm{m}$ ]otivated by the studies of population testosterone levels reviewed earlier, we use the age of 45 years as the cut-off to separate young male CEOs from the rest." (p. 1467). This seems to be a problematic choice to me. First of all, none of the cited studies gives a hint about why the age of 45 should be a particularly meaningful cut point. None of the reviewed studies suggests a significant break or stronger decline of $\mathrm{T}$ at that age. Furthermore, that cut point leads to a very skewed distribution of 16 "young" vs. 341 "old" CEOs.

The strongest reported effect was that of the dichotomized variable "CEO is young" (i.e., younger than 45) on bid withdrawals. Based on the reported descriptive statistics and correlations, it can be computed that 5 young CEOs withdrew, whereas 11 did not. Concerning old CEOs, 40 out of 341 withdrew. This distribution led to the reported Pearson correlation of $r=.12(p=.02)$. If only one young CEO would not have withdrawn, the correlation would have been nonsignificant $(r=.08, p=.13$ ). If only three young CEOs would not have withdrawn, age and withdrawing behavior would be completely unrelated. Hence, as Levi et al.'s conclusions stand and fall on the decision of one single CEO, these results do not seem very robust to me. Why is age categorized at all? Methodological papers on the topic clearly suggest not to dichotomize if a predictor variable can be used on an interval or ratio scale (e.g., Royston, Altman, \& Sauerbrei, 2006).

\section{Conclusion}

Referring to several meta-analyses, it could be shown that the relation between $\mathrm{T}$ and dominant human behavior is much less pronounced than suggested by Levi and colleagues. Likewise, the relation between age and testosterone, especially in the restricted age range of their sample, is presumably close to zero. From my reading of the empirical evidence, the article should be rewritten as "Age and the mergers and acquisitions game," and it should be acknowledged that the age effect, although significant, has a small effect size.

Of course, age is not a psychological variable per se - it is always a proxy for a true causal factor: "And although it is unquestionably useful to find that a phenomenon covaries with age, neither age nor the related variable time is a causal variable; changes occur in time, but not as a result of time." 

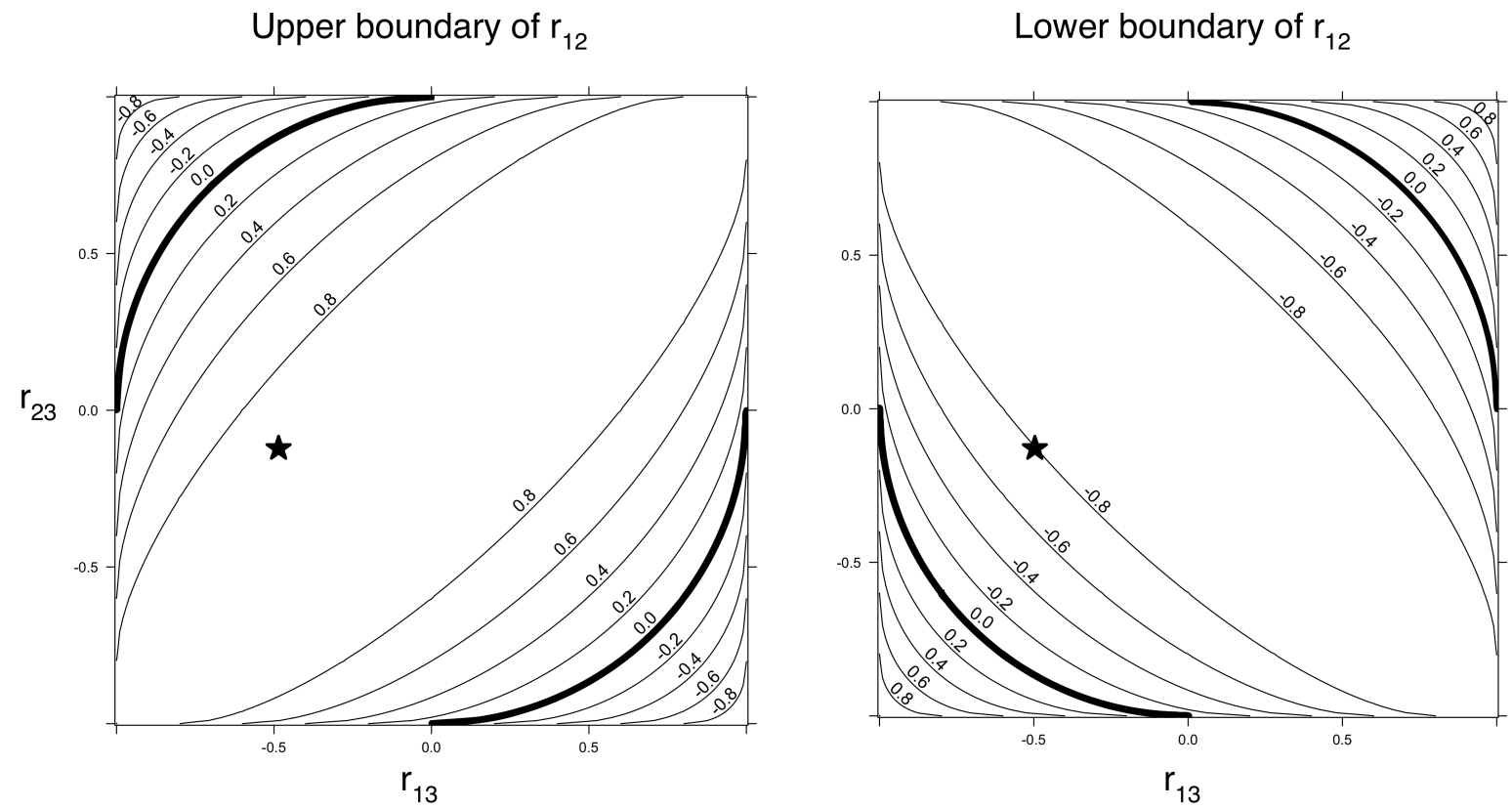

Figure 1. The left panel shows the upper boundary of a correlation $r_{12}$ for the given correlations $r_{13}$ and $r_{23}$, the right panel the lower boundary. The asteriks marks the position of Levi et al.'s study.

(Hartmann \& George, 1999, p. 132). Hence, it would be feasible to spend one or two paragraphs in the discussion speculating about the potential causal role of testosterone (and several other age-related variables). In that article, however, from the main title to the conclusion, the authors argue that $\mathrm{T}$ is the causal factor. Although the authors try to rule out some alternative age-related factors, the main criticism remains: With the current data, there is no way to provide evidence for or against a $\mathrm{T}$ hypothesis. It's simply the wrong story for the data.

Based on the same data set, several other articles could have been written in the same style, for example, "Bicep strength and the M\&A game." Why bicep strength? The theory of embodied cognition predicts that physical dominance is a predictor for psychological dominance in negotiations (Barsalou, 2008). As CEO muscle strength cannot be measured directly, one could use CEO age as a proxy for muscle strength ( $r_{\text {age,musclestrength }}=.55$; Lindle et al., 1997). Physically stronger CEOs, as proxied by age, would therefore be expected to make more dominant choices in M\&As. This ad hoc hypothesis has the same argumentative structure and the same empirical justification as the "testosterone story," and the creative reader can think of a multitude of other agerelated variables that might have an influence on negotiation decisions (e.g., generational cohort effects in moral values, fluid intelligence, different educational levels between age groups, time spent in marriage, or the grayishness of the CEO's hair).

To summarize, Levi et al. conclude that they "[...] have been able to conduct that age appears to be proxying for testosterone rather than experience, horizon, or some other effect" (p. 1478) and that their finding "strongly supports an association between testosterone, as proxied by the bidder male CEO age, and M\&As." (p. 1469). In the light of the analyses given above, that conclusion is not justified.

\section{References}

Archer, J. 2006. Testosterone and human aggression: An evaluation of the challenge hypothesis. Neuroscience \& Biobehavioral Reviews 30(3) 319-345.

Archer, J., N. Graham-Kevan, M. Davies. 2005. Testosterone and aggression: A reanalysis of Book, Starzyk, and Quinsey's (2001) study. Aggression and Violent Behavior 10(2) 241-261.

Barsalou, L.W. 2008. Grounded cognition. Annu. Rev. Psychol. 59 617-645.

Book, A.S., K.B. Starzyk, V.L. Quinsey. 2001. The relationship between testosterone and aggression: A metaanalysis. Aggression and Violent Behavior 6(6) 579-599.

Burnham, T. C. 2007. High-testosterone men reject low ultimatum game offers. Proceedings of the Royal Society B: Biological Sciences 274(1623) 2327-2330.

Cohen, J. 1992. A power primer. Psychological Bulletin 112(1) 155-159.

Ferrini, R. L., E. Barrett-Connor. 1998. Sex hormones and age: a cross-sectional study of testosterone and estradiol and their bioavailable fractions in community-dwelling men. American Journal of Epidemiology 147 750-754.

Gray, A., J. A. Berlin, J. B. McKinlay, C. Longcope. 1991. 
An examination of research design effects on the association of testosterone and male aging: results of a meta-analysis. Journal of Clinical Epidemiology 44(7) 671-684.

Harman, S. M., E. J. Metter, J. D. Tobin, J. Pearson, M. R. Blackman. 2001. Longitudinal effects of aging on serum total and tree testosterone levels in healthy men. Journal of Clinical Endocrinology \& Metabolism 86(2) 724 -731.

Hartmann, D. P., T. P. George. 1999. Design, measurement, and analysis in developmental research. M. H. Bornstein, M. E. Lamb, ed. Developmental psychology: An advanced textbook (4th ed.). Mahwah, NJ US: Lawrence Erlbaum Associates Publishers, 125-195. Levi, M., K. Li, F. Zhang. 2010. Deal or no deal: Hormones and the mergers and acquisitions game. Management Science 56(9) 1462 1483.
Lindle, R. S., E. J. Metter, N. A. Lynch, J. L. Fleg, J. L. Fozard, J. Tobin, T. A. Roy, B. F. Hurley. 1997. Age and gender comparisons of muscle strength in 654 women and men aged 20-93 yr. Journal of Applied Physiology 83(5) 1581-1587.

Olkin, I. 1981. Range restrictions for product-moment correlation matrices. Psychometrika 46(4) 469-472.

Royston, P., D. G. Altman, W. Sauerbrei. 2006. Dichotomizing continuous predictors in multiple regression: A bad idea. Statistics in Medicine 25(1) 127-141.

Wingfield, J. C., R. E. Hegner, A. M. Dufty, G. F. Ball. 1990. The 'Challenge Hypothesis': Theoretical Implications for Patterns of Testosterone Secretion, Mating Systems, and Breeding Strategies. The American Naturalist 136(6) 829846. 